\title{
Infectious Agents in Inflammatory Bowel Disease
}

\author{
P. Rutgeerts, M. Peeters, K. Geboes, G. Vantrappen \\ Department of Medicine and Pathology, University Hospital Gasthuisberg, Leuven, Belgium
}

\section{Early Diagnosis of Inflammatory Bowel Disease}

In the early stage of inflammatory bowel disease it is critical to differentiate between inflammation with a specific cause (Table 1), mostly a gastrointestinal infection, and the onset of ulcerative colitis or Crohn's disease. In the clinical situation microbiological examination of fresh stools is mandatory together with direct examination of rectal slime and depending on the clinical findings also serological tests to detect Yersinia enterocolitica infection or venereal anoproctitis. The initial diagnostic work-up should include ileocolonoscopy, upper gastrointestinal endoscopy and small bowel $x$-ray series. The location of the inflammatory lesions is often an indication of the exact diagnosis (Table 2). During endoscopic examination multiple biopsies have to be obtained in all inflamed areas of the bowel. If endoscopic findings (ileocecal location, short multiple strictures) or clinical findings (lesions on chest $\mathrm{x}$-rays) point towards tuberculosis as a possible cause of the inflammation, Ziehl staining of endoscopic biopsies as well as culture on Löwenstein are necessary.

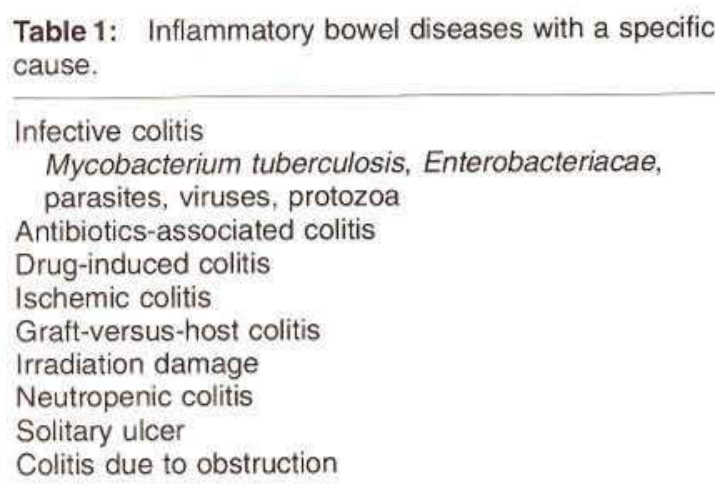

Table 1: Inflammatory bowel diseases with a specific cause.

Infective colitis

Mycobacterium tuberculosis, Enterobacteriacae, parasites, viruses, protozoa

Antibiotics-associated colitis

Drug-induced colitis

Ischemic colitis

Graft-versus-host colitis

Irradiation damage

Neutropenic colitis

Solitary ulcer

Colitis due to obstruction

The examination of biopsies does not make stool cultures obsolete: microbiological studies and histological studies are indeed complementary. The finding of a pathogen is the most important feature in the diagnosis of acute infective (ileo)colitis whereas biopsies are an aid in establishing the diagnosis but very seldom specific. The histological changes are similar in all types of infectious colitis, and as biopsies

Endoscopy 1992; 26: 565-567

(c) Georg Thieme Verlag Stuttgart - New York
Table 2: Etiological diagnosis of ileocolitis related to disease location.

\begin{tabular}{|c|c|c|c|c|}
\hline & Proctitis & Colitis & $\begin{array}{l}\text { Procto- } \\
\text { colitis }\end{array}$ & lleitis \\
\hline \multicolumn{5}{|l|}{ Idiopathic colitis } \\
\hline Ulcerative colitis & + & & + & $(+)$ \\
\hline Crohn's disease & + & + & + & + \\
\hline \multicolumn{5}{|l|}{ Specific colitis } \\
\hline Tuberculous & $(+)$ & + & + & + \\
\hline Infective & + & + & + & + \\
\hline Venereal & + & & $(+)$ & \\
\hline Antibiotic associated & & + & + & \\
\hline Drug-associated & & + & + & \\
\hline Ischemic & $(+)$ & + & $(+)$ & \\
\hline Radiation injury & + & + & + & + \\
\hline \multicolumn{5}{|l|}{ Graft-versus-host } \\
\hline diseases & & + & + & + \\
\hline Obstruction-associatec & & + & & \\
\hline Prolapse-associated & + & & & \\
\hline Solitary ulcer & + & $(+)$ & & \\
\hline Factitious & + & & & \\
\hline
\end{tabular}

from patients with similar clinical manifestations but negative stool cultures have the same histological features the term "acute self-limited colitis" has been introduced.

Optimal interpretation of gastrointestinal biopsies requires that biopsies are obtained from the appropriate site and are in adequate size and number. Biopsies from different sources should be carefully labelled as this information may not only have diagnostic value but be essential if localized abnormalities require additional study by biopsy. In the classification of colitis a distinction must be made between acute and chronic colitis and then maybe between Crohn's disease (CD), ulcerative colitis (UC) and other conditions such as ischaemic colitis for indeterminate colitis. In acute colitis several possible conditions must be considered such as drug induced colitis and inflammation caused by infectious agents. Biopsies are very useful for establishing a differential diagnosis although several problems remain to be solved. The presence of granulomas allows a fairly precise diagnosis as this is characteristic of $\mathrm{CD}$, although tuberculosis must also always be considered especially in Third World countries. In addition, some forms of granulomatous colitis can be induced by uncommon bacteria such as Mycobacterium bovis, even in Europe. Granulomas can also be found 
in other infectious conditions such as schistosomiasis. The finding of an isolated giant cell can point towards CD but this is less characteristic than granulomas and can be seen in other conditions such as an acute infectious colitis and UC and even in normal subjects. Several studies have shown that acute self limited colitis (1), also called acute spontaneously recovering ulcerative colitis (2), can be distinguished from chronic colitis by the presence of a disturbed mucosal architecture. The intraglandular distance, the variabilitiy of the internal glandular diameter and the number of crypts per high-power field are important parameters for making the distinction $(3,4)$. Basal plasmocytosis and crypt distorsion are present at an early stage in UC (5). The ratio of mucosal height to crypt depth is another valuable parameter (6). However, crypt distorsion and a disturbed mucosal architecture are the result of chronic mucosal damage and are therefore not specific for CD or UC. They can indeed occur in other conditions such as Shigella dysentery (7), venereal proctitis (8) and mucosal prolapse syndrome. The presence of a disturbed mucosal architecture allows a presumptive diagnosis of chronic colitis as opposed to acute colitis.

In as many as $21 \%$ of patients with proven inflammatory bowel disease, however, no distinguishing features are present in biopsies taken within three months of the onset of symptoms.

Acute colitis, which is usually the result of an infection, is often referred to as acute self-limited colitis (ASLC). This term has been introduced because patients suffering from this condition recover easily and after a short period but in many of them an infectious agent cannot be identified. Hence "acute infectious colitis" is not an appropriate term. The histological findings in ASLC are a diffuse mucosal oedema, damage of the surface intercryptal epithelial layer and of the upper part of the crypts, mucous depletion, increase of the cellular infiltrate in the lamina propria showing a complex composition and often the presence of a superficial epithelial or mucosal defect. The lamina propria infiltrate may be focal, patchy or diffuse but it is usually more pronounced in the mucosa than in the submucosa. The latter pattern is called "proportionate inflammation". It may however be difficult to distinguish ASLC from a first acute episode of UC or CD. A biopsy diagnosis of ASLC is best made on the basis of absence of histologic features specific for chronic inflammatory bowel disease although in the early phase of UC or CD these features, which mainly indicate chronic mucosal damage, are usually absent. It may therefore be difficult to distinguish an early phase of chronic idiopathic inflammatory bowel disease from a later phase of ASLC. In this later phase of ASLC the cellular infiltrate in the lamina propria is usually composed of more mononuclear cells than in the early phase.

In some forms of infectious enterocolitis the microscopic features may be specific, sometimes resembling those of ischaemic colitis. Such lesions can be seen in Escherichia coli (serotype 0157: H7) induced colitis.

Examination of ileal or colonic biopsies can reveal specificfeatures. Positive Ziehl staining suggests intestinal tuberculosis. Central necrosis of a lymph follicle is highly suggestive of Yersinia enterocolitica infection. Rectal biopsy may help identify the causative agents of colitis if trophozoites of Entamoeba histolytica, acid-fast bacilli or cytomegalovirus or herpes virus inclusions are demonstrated. Findings are also specific in balantidiasis and cryptosporidiosis. Viral inclusions in the presence of inflammatory changes suggest viral (ileo)colitis in the compromised host.

In summary, when a patient presents with bloody diarrhea suggestive of colitis the following steps should be taken:

1) careful documentation of the history, rectosigmoidoscopy with biopsy, repeated stool culture, examination of fresh rectal mucus and serological tests;

2) complete ileocolonoscopy with serial biopsies;

3) antimicrobial chemotherapy if severe symptoms last despite supportive therapy;

4) repeat ileocolonoscopy 3 to 6 months after the acute episode: total healing confirms the diagnosis of acute (infective?) ileocolitis; if lesions persist the patient probably has chronic idiopathic inflammatory bowel disease.

\section{Intercurrent Infections and Relapses of Inflammatory Bowel Disease}

Infections with Enterobacteriacecae may cause a relapse of previously inactive Crohn's disease and ulcerative colitis. In a four-year survey we observed 12 patients with a flare-up of inflammatory bowel disease associated with Yersinia enterocolitica in six cases; Salmonella was isolated in four cases, Campylobacter in one case and cytomegalovirus in one case (9). The attack was severe in all but three patients and toxic colonic dilatation occurred in four patients. It has also been suggested that intercurrent viral infection $(10)$ and overgrowth of the colonic flora with Clostridium difficile may cause flare-ups of inflammatory bowel disease $(11,12)$.

Following the report of Lamont et al. (11) most gastroenterologists screen all patients with acute flare-ups of inflammatory bowel disease for Clostridium difficile and its toxins with few positive results. Expensive therapy with vancomycin perorally does not seem to be effective in controlling these relapses of inflammatory bowel disease and the relevance of detecting Clostridium difficile or its toxins can be questioned.

A number of features suggest a relationship between infections of the gastrointestinal tract and chronic idiopathic inflammatory bowel disease. It seems established that in areas with a high incidence of acute infective enterocolitis ( $\mathrm{Sal}$ - 
monella typhi, Shigella dysenteriae, Entamoeba histolytica) the incidence and prevalence of inflammatory bowel disease is low. Moreover, in a fairly significant number of patients the onset of inflammatory bowel disease coincides with a documented intestinal infection. At the cellular level a lot of similarities exist between infective colitis and inflammatory bowel disease. Expression by epithelial cells of MHCclass II antigens is present not only in inflammatory bowel disease but also in infective colitis. Experimental findings in rats show that this expression can disappear with the infection and return with reinfection (13). The mechanisms by which infections could cause chronic inflammation are unknown but they might trigger off autoimmune phenomena by upregulation of the immune system, by delayed immunologic reaction caused by molecular mimicry or even by alteration of host antigens or exposure of hidden antigens.

\section{Etiopathogenesis of Inflammatory Bowel Disease}

The resemblance between Crohn's disease and intestinal tuberculosis has stimulated interest in mycobacterial infection as the cause of Crohn's disease. Positive cultures for mycobacteria ( 1 of 27) or spheroplasts (22 of 27) from mesenteric lymph nodes in patients with Crohn's disease reported by Burnham et al. (14) greatly stimulated this interest.

More recently a previously unrecognized Mycobacterium species resembling Mycobacterium paratuberculosis was isolated from mucosal tissue of patients with Crohn's disease $(15,16)$. Culture of endoscopic mucosal biopsies have also yielded growth of mycobacteria (17). However, the data remain controversial, especially since all serological studies are inconclusive. Widespread culture of mucosal biopsies in Crohn's disease therefore does not seem indicated. Trials with antimycobacterial chemotherapy are currently underway.

Findings in other microbiological studies may be relevant in the etiopathogenesis of Crohn's disease. An important study was conducted by Heidt et al. (18). In endoscopic small bowel biopsies in 14 of 28 patients with chronic idiopathic enterocolitis these authors identified monospecific antibodies against Yersinia enterocolitica with $\operatorname{IgA}$ antibodies against plasmid encoded proteins of Yersinia enterocolitica in eight patients. Further research is certainly indicated.

\section{References}

1. Kumar NB. Nostrant TT. Appelman HD: The histopathologic spectrum of acute self-limited colitis (acute infectious type colitis). Am. J. Surg. Pathol. 1982: 6: 523-529.

2. Bonfils $S$, Hervoir P, Girodet $J$ et al: Acute spontaneously recovering ulcerating colitis (ARUC). Report of 6 cases. Am. J. Dig. Dis. 1977: 22: $429-436$.

3. Rubio CA. Johansson C. Kock $Y$ : A quantitative method of estimating inflammation in the rectal mucosa. III. Chronic ulcerative colitis. Scand. J. Gastroenterol. 1982: 17: 1083-1087.

4. Rubio CA. Johansson C. Uribe A. Kock Y: A quantitative method of estimating inflammation in the rectal mucosa. IV. Ulcerative colitis in remission. Scand. J. Gastroenterol. 1984: 19:525-530.

5. Nostrant TT, Kumar NB. Appelman HD: Histopathology differentiates acute self-limited colitis from ulcerative colitis. Gastroenterology 1987; 92: 318-328.

6. Jenkins D, Goodall A, Drew B, Scott BB: what is colitis? Statistical approach to distinguishing elinically important inflammatory change in rectal biopsy specimens. J. Clin. Pathol. 19888: 41: $72-$ 79 .

7. Anand BS, Malhotra V. Bhattacharya SK et al.: Rectal histology in acute bacillary dysentery. Gastroenterology 1986: 90: 654-660.

8. Suravicz CM, Goodell SE, Quinn TC et al.: Spectrum of rectal biopsy abnormalities in homosexual men with intestinal symptoms. Gastroenterology 1986; 91; 651-659.

9. Rutgeerts $P$, Geboes $K$. Ponette $E$ et al.: Acute infective colitis caused by endemic pathogens in Western-Europe: Endoscopic Features. Endoscopy 1982: 6: 212-219.

10. Gebhard, Greenberg HB. Singh $N$ et al.: Acute viral enteritis and exacerbations of TBD. Gastroenterology 1982: 83: 1207-1209.

11. LaMont T, Trnka YM: Therapeutic implications of Clostridium difficile toxin during relapse of chronic inflammatory bowel disease. Lancet 1980); 23: 381-384.

12. Bolton RP. Reud AE: Clostridium difficile in toxic megacolon complicating acute inflammatory bowel disease. Br. Med. J. 1982; 285: $475-476$

13. Masson SD, Perdue MH: Changes in distribution of la antigen on epithelium of the jejunum and ileum in rats infected with Nippostrongylus brasiliensis. Clin. Imm. Immunopath. 1990; 57: 83-95.

14. Burnham WR, Lennand-Jones IE. Stanford JI. Bird RG: Mycobacteria as a possible cause of inflammatory bowel disease. Lancet 1978: ii: $693-696$.

15. Chiodini R.J. Van Kruiningen HJ. Thayer WR et al.: Possible role of mycobacteria in inflammatory bowel disease. An unclassified mycobacterium species isolated from patients withCrohn's disease. Dig. Dis. Sci. 1984; 29: 1073-1079.

16. Chiodini RJ, Van Kruiningen HJ. Thaver WR et al: Mycobacterial spheroplasts isolated from patients with Crohn's disease, Gastroenterology 1986: 88: 1348-1352.

17. Colemont LJ. Pattijn SR. Michielsen PP et al.: Acid-fast facilli in Crohn's disease, Lancet (Letter) 1988: 1: 294-295.

18. Heidt $H$. Kanch $H$. Arndt $R$ et al.: Mikrobiologische Befunde bei protrahiert verlaufenden und chronischen Enterocolitiden. Endoskopie Heute 1991; 1: 28-31.

Corresponding Author

Prof. P. Rutgeerts, Department of Medicine and Pathologie, University Hospital Gasthuisberg, 3000 Leuven, Belgium 